\title{
Creating Environmental Awareness in Service Oriented Software Engineering
}

\author{
Patricia Lago ${ }^{1}$ and Toon Jansen ${ }^{2}$ \\ ${ }^{1}$ VU University Amsterdam, The Netherlands \\ patricia@cs.vu.nl \\ ${ }^{2}$ Het Expertise Centrum, The Netherlands \\ T.Jansen@hec.nl
}

\begin{abstract}
Carbon emission of IT is an issue. ICT energy consumption is expected to grow by $73 \%$ (instead of the originally targeted 26\%) until 2020, and the service sector alone counts for $70 \%$ of the European economy. Energy consumption is a combination of what we use, and how we use it. Most green initiatives look at what types of devices do consume energy, and try to optimize their up-time as such. Few initiatives, though, measure how do software systems actually use these devices, with the goal of optimizing consumption of devices and computing resources. Basic research is needed to address this software optimization problem. The proposed approach is to make visible the environmental impact of software services by measuring it. In this way, we will become aware of the amount of energy needed by our software, and hence learn how to target software optimization where it is mostly needed. As a first step in this direction, in this paper we define three main problem areas to realize green service-based applications, and propose a service-oriented approach to address them. Thanks to that we can bring clarity in what entails managing and developing green software according to environmental strategies.
\end{abstract}

Keywords: energy-efficient software, green IT, service orientation, green metrics, sustainability.

\section{Introduction}

In the last decade, Service Oriented Software Engineering (SOSE) and ServiceOriented Computing have emerged as a major software development discipline resembling (if not outbalancing) what object orientation meant for the IT market of the eighties. Service Oriented Architecture (SOA) captures a logical way of providing software services both within an enterprise, and across organizational and national boundaries to either end-user applications or other services distributed on a network [3]. A well-constructed SOA can empower a business or social environment with a flexible infrastructure and processing environment by providing independent, reusable automated business processes (as services), and a robust foundation for leveraging these services $[2,6]$.

Due to its fast application in all aspects of our society, SOSE should address environmental issues promptly: in this period of acceptation and learning, we should think 
about how to sensitize people to the adoption of an ecological approach to the construction, deployment and use of service-based applications (SBAs). Unfortunately, service-oriented software is often developed assuming availability of unlimited resources: 24x7 service availability, unlimited use of computing power, hardware, network and printing resources (all demanding energy and causing carbon emission). The increase in the network traffic and in the number of electronic data centers is having a huge impact on the environment (an average data center is consuming the same amount of energy as about 25,000 households [9]). According to recent Gartner estimates ${ }^{1}$, ICT industry accounts for approximately 2 percent of global carbon dioxide (CO2) emissions, a figure equivalent to aviation. Almost $90 \%$ of young Americans are "always connected". This also impacts energy consumption, e.g., in the Netherlands, total electricity consumption increased between 2006 and 2008 by $12 \%$. Without focused environmental strategies, ICT related energy consumption is expected to grow by $73 \%$ until 2020 (instead of the targeted 26\%).

IT systems and data centers are migrating towards a service-oriented approach in which the available computing resources are shared by several types of users and organizations. In such systems, the software is accessed as-a-service and computational capacity is offered on demand to customers who share a pool of IT resources in-the-cloud [5]. The software as-a-service (SaaS) model provides significant economies of scale, affecting the energy efficiency of data centers [1].

Environmental strategies are already a reality in non-IT domains. For instance, Sweden recently introduced on many supermarket products an indication of the $\mathrm{CO} 2$ emission related to their production process [8], to change alimentary habits toward more environmental friendly products, similar to what already happens for choosing cars and house-hold equipment. As another example in the more traditional hardware/embedded software domain, some companies already commercialize devices that are plugged in the electricity socket and allow users to monitor and gain detailed understanding of their energy consumption.

Adoption is much more difficult in intangible domains like software industry for at least two reasons. First, it is a challenge to increase process awareness, i.e. convince decision makers of the urgency of adopting ecological strategies in the processes of managing their IT portfolios and bring IT practitioners to adopt innovative ecological models in the way they engineer SBAs, i.e. in the development process. Second, we urgently need to change the way users exploit SaaS, by making them realize their energy consumption and suggest alternative consumption models. I.e. we need to create people awareness. Third, it is difficult to make explicit how SBAs should be engineered to become "greener", i.e. to define and implement how ecological strategies can be adopted by service-oriented software (service awareness). Awareness can be increased with a SaaS model, by offering "green metrics" to measure the level of greenness of software services, and incorporate in SBAs ready-to-use "feedback services", i.e. services that give feedback on the carbon footprint of SBAs and of their end-users.

In this paper we describe how service-oriented software can positively influence the software impact on the environment, i.e. we introduce the concept of green problem areas, defined as green issue categories that have to be resolved to achieve energy efficient software services and SBAs.

\footnotetext{
${ }^{1}$ Source: www.gartner.com/it/page.jsp?id=503867 [15 September 2010].
} 
As a result of literature scan and discussions with both researchers and practitioners in the field, we have distilled three green problem areas (further discussed in Section 2): the direct environmental impact of executing software services (service awareness); the indirect environmental impact of their development process (process awareness); and the use of services to monitor and measure the two above, so to create people awareness). Thanks to this holistic overview of green problem areas in SO we devised an approach, called Service Greenery, to address them in a structured way, as presented in Section 3 and based on previous work [4]. Conclusions and directions for future research conclude the paper.

\section{Green Problem Areas in Service Oriented Software Engineering}

Research efforts are needed to investigate the role of software services in making enterprises "greener". To this end, business strategies decided at the enterprise level must be aligned with green strategies (as illustrated in Fig. 1). Only in this way ecologic issues are incorporated in the strategic plan of the organization and transformed into business opportunities.

Green strategies can address three green problem areas, as illustrated in the Figure:

- Create process awareness by having a more sustainable (or agile) development process. Green strategies should influence (cf. Fig. 1) the process

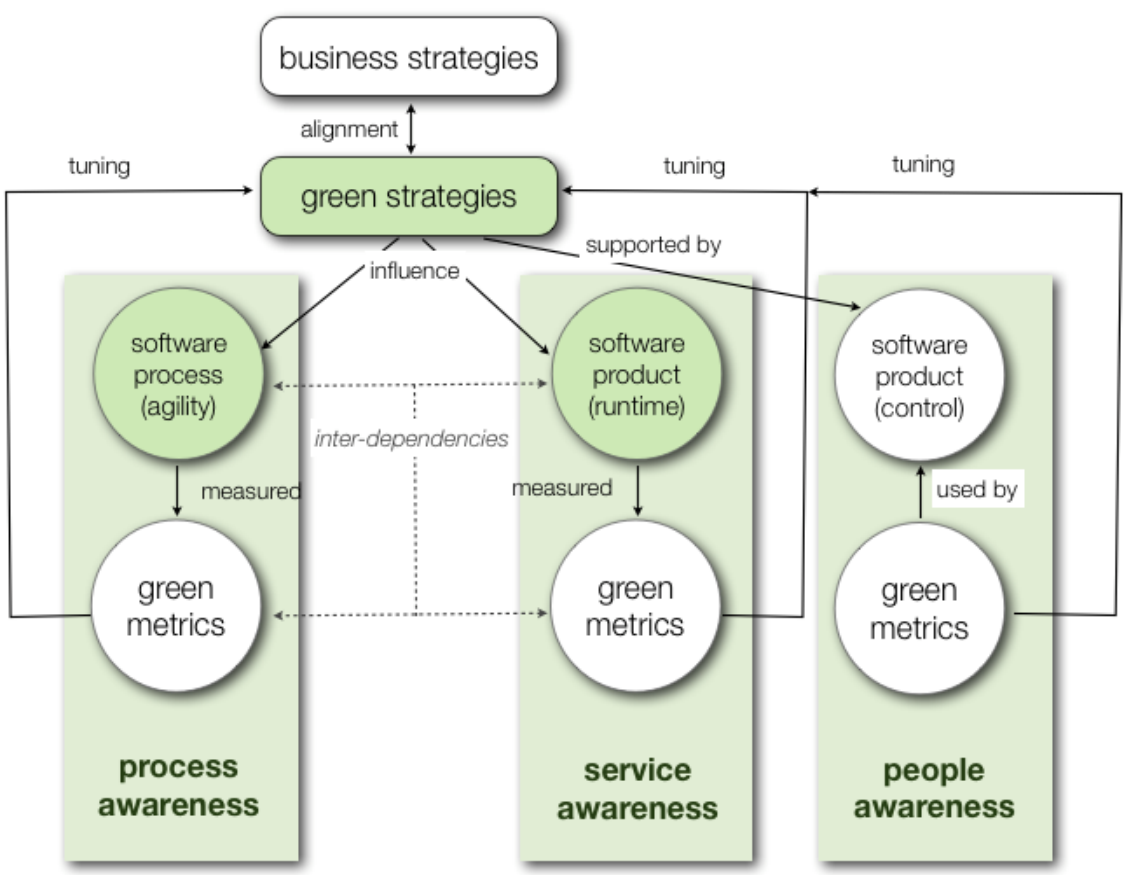

Fig. 1. Green Problem Areas in Service Orientation 
followed to develop software services and SBAs (i.e. software process). By defining suitable green (process) metrics we can measure the current level of greenness of the processes, and tune the related green strategies to optimize it, making sure to achieve the target environmental objectives of the organization.

- Create service awareness by delivering energy-efficient software services and SBAs. Green strategies should influence (cf. Fig. 1) the direct impact of executing the software services/SBAs to render them energy-efficient and hence lower their own carbon footprint at runtime. To create service awareness green (service) metrics provide the necessary feedback to measure the direct environmental impact of software services and SBAs.

- Create people awareness by putting in place software especially conceived to control the impact of IT and its actors on environmental issues. Green strategies can be supported by (cf. Fig. 1) SBAs which are used to control some eco-friendly systems (e.g. SBAs controlling smart grids), to control the impact of individuals (e.g. SBAs regulating energy consumptions in smart homes), or to create awareness about some environmental issues (e.g. printing budgeting). In the first two cases we need to measure the process (respectively product runtime) impact on the environment (by use of especially conceived green metrics). To create people awareness, SBAs can use green metrics to realize the decided upon green strategies.

In summary, results of measurements can inject a tuning process to evolve the green strategies of an organization, and eventually determine the adaptation of the business strategies. The ability to define sound green metrics for process, service and people awareness is essential to realize this green vision. While some work is being done in this respect (e.g. [1]), most metrics address the environmental impact of IT rather than the software/services using it [7]. We regard the definition and experimentation of green metrics for SOSE in the three green problem areas as an important and mostly unexplored research area.

\section{The Service Greenery Approach to Green SBAs}

To increase both process, service and people awareness we propose an approach to create what we call service greenery, i.e. a portfolio of green software services accessible on the Web as-a-service. The service greenery supports the iterative, incremental approach to increasing sustainability, as illustrated in Fig. 2. This is centered around two types of services (central part in the Figure):

- Environmental strategies as-a-service, which set out the user's/organization's goals and actions for achieving a sustainable environment. Examples of generic organizational strategies are 'achieve tighter customer relationships' or 'become more standard'. Equivalent green strategies could be 'achieve carbon neutral data traffic' or 'decrease the company's global carbon footprint by $\mathrm{x} \%$ before year y'. Such strategies will be defined in terms of the user's/organization's business processes. Accordingly, users/organizations can use environmental strategies as instrument for lowering carbon footprint, and at the same time keep them aligned with the supporting IT services/SBAs. 
- Green metrics as-a-service, which measure the actual carbon footprint of SBAs. In this way, the user/organization can monitor real-time the SBA environmental impact and provide feedback to end-users and companies. This will increase people awareness (by leading users to opt for options consuming less energy) as well as service awareness (by quantifying the current carbon footprint).

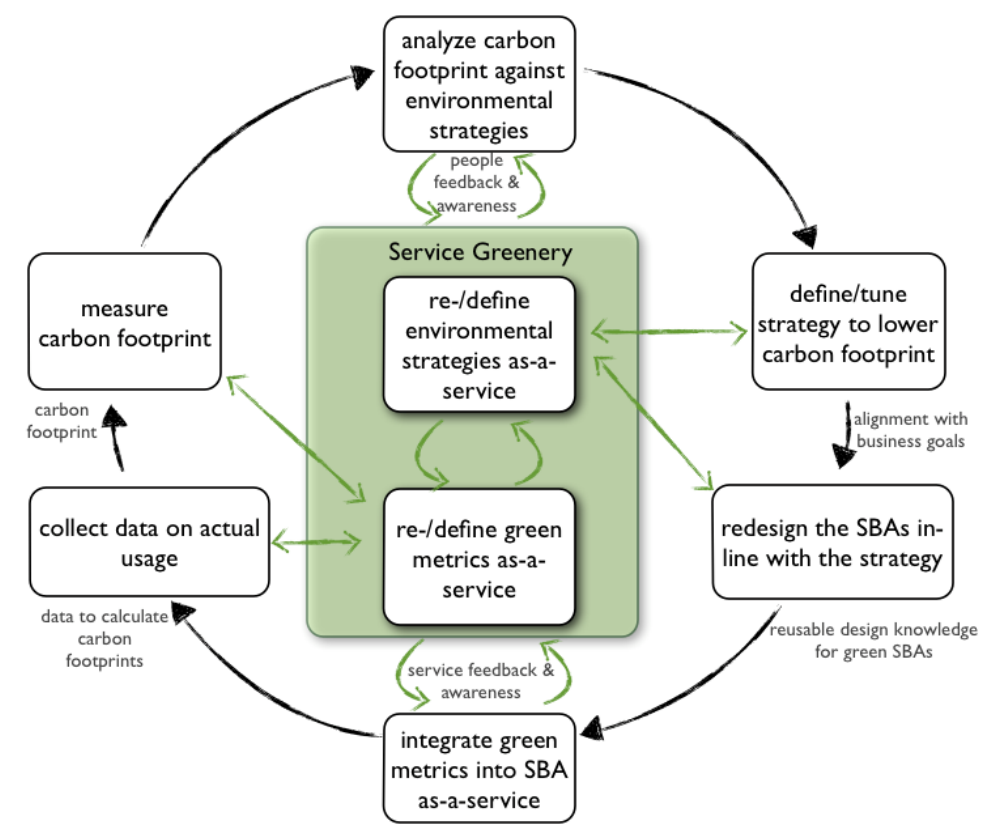

Fig. 2. Central role of the Service Greenery to redesign environmental-aware SBAs

- The service greenery will make available environmental strategies as-a-service and their supporting green metrics as-a-service. It will trigger use and reuse of the green metrics, create incentives to tailor strategies and measures to business goals, hence re-populating the service greenery with new resources. The ultimate goal is to make service oriented software "greener" by initiating the adoption of environmental strategies offered as-a-service, continuously measuring the achieved level of greenness, and tune the strategies to increase it.

\section{Conclusions and Research Directions}

In this paper we introduce three problem areas that frame the issue of creating environmental awareness in SOSE: the creation of process awareness, service awareness and people awareness. We also give a holistic overview of how enterprise business strategies and green strategies should be aligned. Green strategies influence process and service awareness, and the application of green metrics trigger the tuning of the 
strategies. People awareness supports the achievement of green (and hence business) strategies thanks to the use of green metrics creating such awareness.

To address the green problem areas we also proposed an approach based on the SaaS model. The service greenery should offer a portfolio of services to measure relevant factors in the SOSE process and the level of greenness of software services/SBAs, as well as create people awareness.

No need to say that by making the ingredients of the service greenery available as reusable assets we can identify innovative ways to greenify the industry IT portfolio; we can increase process-, service- and people awareness thanks to continuous measurement \& feedback; we can disseminate reusable green knowledge offered to researchers and practitioners.

While the three green problem areas could hold for any software, too, we study them specifically for service-oriented software, as more and more enterprises are migrating their software assets to SOA technologies. As future work we will study further the green factors belonging to each area, and their dependencies. This will help us in defining the relevant related green metrics.

Acknowledgments. This research has been partially funded by the European Community's Seventh Programme FP7/2007-2013 under grant agreement 215483 (SCube).

\section{References}

1. Ferreira, A.M., Kritikos, K., Pernici, B.: Energy-Aware Design of Service-Based Applications. In: Baresi, L., Chi, C.-H., Suzuki, J. (eds.) ICSOC-ServiceWave 2009. LNCS, vol. 5900, pp. 99-114. Springer, Heidelberg (2009)

2. Gu, Q., Lago, P.: Exploring Service-Oriented System Engineering Challenges: A Systematic Literature Review. Journal of Service Oriented Computing and Applications 3, 171-188 (2009)

3. Lago, P.: Establishing and Managing Knowledge Sharing Networks. In: Ali Babar, M., Dingsoyr, T., Lago, P., van Vliet, H. (eds.) Software Architecture Knowledge Management - Theory and Practice, pp. 113-131. Springer, Heidelberg (2009)

4. Lago, P., Jansen, T., Jansen, M.: The Service Greenery - Integrating Sustainability In Service Oriented Software. In: International Workshop on Software Research and Climate Change (WSRCC), co-located with ICSE, 2 pages (2010)

5. Dikaiakos, M.D., Katsaros, D., Mehra, P., Pallis, G., Vakali, A.: Cloud Computing: Distributed Internet Computing for IT and Scientific Research (Guest Editors' Introduction). IEEE Internet Computing 13, 10-13 (2009)

6. Papazoglou, M.P.: Web Services: Principles and Technology. Prentice-Hall, Englewood Cliffs (2007)

7. Gude, S., Lago, P.: A Survey of Green IT - Metrics to Express Greenness in the IT Industry, Technical report VU University Amsterdam (August 2010)

8. Rosenthal, E.: To Cut Global Warming, Swedes Study Their Plates. New York Times (October 22, 2009)

9. Kaplan, J.M., Forrest, W., Kindler, N.: Revolutionizing Data Center Energy Efficiency McKinsey \& Company (August 2008) 